REVISTA DE URBANISMO

ISSN 0717-5051

http://revistaurbanismo.uchile.cl
Revista de Urbanismo N40 | Junio 2019

Departamento de Urbanismo | FAU | Universidad de Chile

\title{
El nuevo proyecto de Av. Perú de Recoleta como espacio de integración urbana
}

Perú St. in Recoleta, Santiago de Chile, as a space for urban integration

\section{Paulina Ahumada*, Juana Zunino** y Paz Carreño**}

Recibido: 31 de marzo de 2018

Aceptado: 01 de abril de 2019

\section{Resumen}

El presente artículo presenta el caso de un proyecto de espacio público en la Comuna de Recoleta, Santiago de Chile, que consolida y genera un parque longitudinal que integra distintas escalas urbanas y grupos sociales.

Poniendo el foco en los problemas y desafíos de la integración urbana, el texto revisa la evolución histórica y el proceso de nueve años del proyecto, proponiendo un análisis de dos esferas de la integración: la integración socioespacial y los desafíos de la integración sectorial y de los distintos niveles de toma de decisiones involucrados en una intervención urbana.

El análisis aborda la etapa de planificación y la formulación de encargo del proyecto; la gestión urbana desde el nivel local; y, la estrategia de diseño del espacio público para lograr un diseño integral y una propuesta paisajística que valora los atributos naturales del lugar, incorporando el uso de flora nativa que aún está en estado incipiente en nuestro país.

Palabras clave: espacio público y equidad territorial, gestión urbana, integración urbana, paisajismo y flora nativa.

\begin{abstract}
This article presents the case of a public space project in Recoleta, Santiago de Chile, which consolidates and generates a longitudinal and integrated green spatiality of different urban scales and social groups.

Focusing on the problems and challenges of urban integration, the text reviews the historical evolution and the 9year process of the project, proposing an analysis of two areas of integration: socio-spatial integration and the challenges of sectoral integration and of the different levels of decision making involved in an urban intervention.

The analysis addresses the planning stage and the commission formulation of the project; urban management from the local level; and, the design strategy of the public space to achieve an integral design and a landscaping proposal that values the natural attributes of the place, incorporating the use of native flora that is still incipient in our country.
\end{abstract}

Keywords: landscaping and native flora, public space and territorial equity, urban integration, urban management.

*Filiación: Universidad de Santiago de Chile, Santiago, Chile. Contacto: pahumada@recoleta.cl

**Filiación: Santiago, Chile. Contacto: juanazunino@gmail.com, paz.carreno@gmail.com

Cómo citar: Ahumada, P., Zunino, J. y Carreño, P. (2019). El nuevo proyecto de Av. Perú de Recoleta como espacio de

integración urbana. Revista de Urbanismo, 40, 1-20. https://doi.org/10.5354/0717-5051.2018.48837




\section{Introducción}

El problema de la integración urbana es un tópico importante dentro de la discusión académica y de las políticas públicas sobre la ciudad. En las últimas décadas la segregación socio espacial se ha evidenciado como un problema que afecta transversalmente a ciudades de distintas latitudes y de distintas escalas. En este marco es interesante ver los desplazamientos que se han ido produciendo en el uso del concepto integración, lo cual es una muestra de los distintos enfoques y aristas del problema.

En un primer momento, el foco se ubica en el estudio de la segregación socioeconómica y espacial, lo que luego fue derivando hacia el uso del vocablo integración, en la medida en que la discusión fue pasando del diagnóstico a la búsqueda de soluciones. En ese desplazamiento, resulta clave entender el cambio en las escalas de análisis, el cual pasa de hacer estudios generales de la ciudad a estudios a nivel de barrios, desde los cuales se rebaten los mitos sobre la segregación y se observa en los distintos grupos socioeconómicos niveles de disposición a la integración (Sabatini, Cáceres y Cerda, 2001; Sabatini y Brain, 2008; Sabatini, Mora y Polanco, 2013; Sabatini y Vergara, 2018)

A su vez, al pasar de los diagnósticos a las soluciones, el concepto de integración aparece ligado a otro ámbito: el de la gestión urbana, como el espacio físico e institucional donde se debate la forma de abordar y resolver los problemas de integración urbana.

El nuevo proyecto de espacio público de la Av. Perú en la Comuna de Recoleta es una intervención que se ubica en ese cruce entre los problemas de integración social y los desafíos de la integración ligada a la gestión, en este caso en una escala municipal y de barrio. Desde esta ubicación particular este artículo presenta el caso y realizar un análisis del proceso de desarrollo del proyecto a partir de la siguiente pregunta de investigación: ¿qué se puede aprender sobre problemas y soluciones de integración urbana desde el caso de un proyecto de espacio público de escala municipal?

El proyecto posee un conjunto de características que impactan diversas escalas y grupos sociales a través del desarrollo de su trazado y elementos urbanos, donde un desafío estratégico fue la integración urbana. En particular, busca consolidar un área verde preexistente en un barrio tradicional de inmigrantes árabes, que hoy se ha visto renovado, y dotar de una nueva área verde a un sector vulnerable, con el fin de lograr un cambio en el entorno y su uso. Considera una ciclovía que se integra al plan metropolitano de ciclo rutas. El eje que se interviene conecta los barrios de Bellavista y de Cementerios con un nuevo proyecto hito (el Parque de la Infancia, de Elemental) y a la vez bordea el Cerro San Cristóbal, lo que es una particularidad geográfica que incide en la valorización de los atributos naturales del lugar y las opciones del uso de flora nativa.

La obra fue inaugurada en febrero de 2018 , como culminación de un proceso, que abarca un período de casi 10 años, desde la formulación de la idea a la construcción de la obra urbana. Un período que, desde la inmediatez contemporánea, resulta excesivo. Sin embargo, se considera más común de lo que parece en los tiempos de las intervenciones urbanas.

Es por esto por lo que, lo importante es mostrar la obra centrándose en particular en una relectura o revisión del proceso y los desafíos planteados de integración urbana. Un proceso que comprende la realización de dos concursos públicos de diseño, la etapa final que comprende el diseño arquitectónico, paisajístico y de especialidades, la gestión de la iniciativa desde un municipio, la obtención de financiamiento estatal, la participación ciudadana, un trabajo coordinado con los servicios de nivel central y, por último, la construcción misma.

Como señala Busquets (2011), entendemos el urbanismo como un saber práctico, un desafío es el cómo aprendemos de esas prácticas. Es un hecho que las prácticas de intervenciones urbanas realizadas desde el sector público no son un objeto común de sistematización e investigación desde el lugar mismo del quehacer público. Sin embargo, los recientes estudios sobre el caso de Proyecto Urbano Ribera Norte de Concepción proponen un camino a seguir (Salinas y Baeriswyl, 2017).

El artículo se divide en cinco partes, donde se presenta primero el marco teórico y metodología propuesta, para luego presentar y analizar tres momentos y aspectos del proceso: el paso de la planificación al proyecto; luego la gestión urbana desde 
el nivel local y, por último, se presenta la estrategia de diseño del espacio público como un desafío de diseño integral e integración urbana.

\section{Problemas de integración: de la integración socioespacial a la integración intersectorial y multiescalar}

Los problemas de segregación residencial urbana son un tópico relevante dentro de los estudios sobre la ciudad en las últimas décadas. Las políticas urbanas neoliberales, instaladas en Chile desde los 1990, establecieron como un foco la superación de la pobreza y los déficits de vivienda a través de la producción masiva de esta misma y su acceso y financiamiento a través de un sistema de voucher. Esta política aparentemente exitosa, instalada en Chile y en otros países de América Latina, "terminó creando nuevos problemas urbanos y sociales: segregación, fragmentación, inseguridad, hacinamiento" (Rodríguez y Sugranyes, 2004, p.53).

La ciudad construida bajo las lógicas de la liberalización del mercado del suelo urbano, la desregulación y ausencia de planificación han dado como resultado crecientes problemas de segregación urbana, que en las últimas dos décadas han sido estudiadas para Chile y Latinoamérica por varios autores (Sabatini, Cáceres y Cerda, 2001; Rodríguez y Arriagada, 2004; Sabatini y Brain, 2008; Sabatini, Mora y Polanco, 2013; Sabatini y Vergara, 2018). Se levanta así la evidencia de que la pobreza urbana se debate entre el acceso a la vivienda y el acceso a la ciudad o a los bienes y servicios urbanos. Es evidente la existencia de un acceso desigual a los servicios urbanos, en primer término, reflejado en los problemas de movilidad y accesibilidad y luego en relación con otros servicios urbanos, como es el caso de la desigual distribución dentro del territorio de áreas verdes y espacios público de calidad (Reyes y Figueroa, 2010).

Un aspecto interesante de los estudios sobre segregación, entendida "como el grado de proximidad espacial o de aglomeración territorial de las familias pertenecientes a un mismo grupo social" (Sabatini, Cáceres y Cerda, 2001, p.27), son las diferencias que se registran al estudiar el fenómeno a distintas escalas. A una escala de ciudad se evidencian grandes áreas claramente diferenciadas, donde residen las familias de niveles socio económicos altos (en el caso de Santiago las comunas del cono oriente) en contraste con las comunas periféricas (del área sur, poniente y norte) que concentran los residentes de más bajos ingresos. En cambio, a un nivel intraurbano y de barrios se observan progresivos grados de integración entre familias de distintos niveles socioeconómicos, rebatiendo con ello los mitos sobre las dificultades estructurales que tendría la integración social (Sabatini y Brain, 2008).

Hay que destacar el desplazamiento que se observa en el tiempo del uso del concepto segregación al uso del concepto integración. La palabra segregación se usa asociada al diagnóstico del problema, en tanto el uso del concepto integración aparece en los estudios más recientes y asociado a las acciones para solucionar el problema (Sabatini, Mora y Polanco, 2013). Es así como en Chile se ha producido un cambio en la política pública sobre vivienda social que se refleja en la aparición del programa y subsidio de vivienda integrada (MINVU).

En este marco se plantea el estudio de los beneficios que pueden tener para la integración el apoyo a las personas (vía voucher) o el apoyo a los lugares, que significa el desarrollo de proyectos integrales donde la vivienda social es parte de un conjunto de inversiones en infraestructura, áreas verdes y espacios públicos localizados en un barrio o sector estratégico (Sabatini y Vergara, 2018).

En este giro que significa el cambio de las personas a los lugares, aparece en la literatura otro ámbito donde se discute sobre integración, pero referida ahora a los desafíos de la gestión de proyectos urbanos.

La gestión, entendida en términos simples "como la manera de hacer que las cosas ocurran" (Moris, 2009, p.1) supone según el mismo Moris (2009) "la necesidad de contar con instrumentos que promuevan la integración intersectorial y multiescalar" (p.2).

No es casual que tanto en el estudio de Sabatini (2018) como en el de Moris (2009) se refieran a la experiencia del desarrollo del Proyecto Urbano Ribera Norte de Concepción. Un proyecto que surge desde la experiencia regional y local, que se desarrolla en el largo plazo (20 años) y que supone enfrentar todos los conflictos ligados a una gestión compleja: multisectorial y que involucra diferentes escalas de decisiones. Ribera Norte representa un interesante estudio de caso (Salinas 
y Baeriswyl, 2017; Zunino, 2005; Moris, 2009) en el cual se han abordado los problemas de la gestión ligados a todo el ciclo de vida de un proyecto; a la participación de distintos actores, partiendo por las familias residentes de los campamentos hasta los alcaldes y autoridades de todo el período; a los desafíos de la coordinación institucional entre las instancias sectoriales (vivienda, transporte, infraestructura, etc.) y los niveles jerárquicos comunal, regional y nacional.

Así como el cambio de escala sirvió para mirar desde una nueva perspectiva el problema de la segregación y pasar del concepto de segregación al de integración urbana, en este artículo se propone también un cambio de escala: analizar cómo caso de estudio la experiencia de un proyecto de intervención de espacio público de nivel comunal. Un cambio de escala enfocado a mirar desde esta nueva perspectiva los temas de integración social, intersectorial y multiescalar. Mirar desde abajo, desde la escala del barrio y de lo local, pero sin perder de vista el paso de lo local a lo global.

En este sentido la pregunta de investigación que plantea el artículo es ¿qué se puede aprender sobre problemas y soluciones de integración urbana desde el caso de un proyecto de espacio público de escala municipal? Se propone para ello, registrar en forma sistemática una experiencia de 9 años, y evaluar los aprendizajes que pueda entregar respecto de los dos ámbitos de integración presentados: la integración social, en un barrio y los desafíos de la integración intersectorial y multiescalar que supone un proyecto de espacio público.

\section{Metodología}

El enfoque metodológico usado corresponde al estudio de caso, que realiza una presentación descriptiva del caso, considerando en especial distinguir los "factores (que) pertenecen a la esfera de lo técnico, lo institucional, lo político y lo social" (Echeverri y Orsini, 2011, p.21). Se presenta, por lo tanto, un registro del proceso, con especial énfasis en dar cuenta de los actores involucrados y la toma de decisiones claves, para luego hacer una revisión y lectura crítica del mismo.

La presentación del caso se divide en tres momentos y aspectos claves, los cuales son:
Siguiendo la huella desde la planificación al proyecto, que busca dar cuenta de la génesis y del cómo se explica la progresiva emergencia y concreción de este caso, en un territorio determinado de la ciudad y la comuna, en un contexto de una dinámica urbana particular y de momentos políticos que lo marcan.

La gestión urbana desde el nivel local, donde se presenta la evolución de la iniciativa desde el lugar particular que significa la escala municipal, como una escala intermedia entre lo local y ciudadano y las políticas urbanas de nivel central.

Por último, se expone el proyecto mismo de diseño de un espacio público como un desafío de diseño integral e integración urbana, donde los ejes están en la noción de espacio público, por sobre los enfoques sectoriales de vialidad o movilidad, y la propuesta paisajística que es una apuesta por valorizar la flora nativa y mediterránea a partir de la vegetación del lugar.

\section{Siguiendo la huella desde la Planificación al Proyecto}

Según la argumentación de Moris (2009) un elemento importante del desafío de integración es el nexo entre la gestión y la planificación. En este caso el eje vial Av. Perú aparece en el primer plan regulador de Santiago (Brunner, 1932). A su vez como barrio, es un territorio que corresponde a los márgenes de la ciudad propia, los extramuros de La Chimba, que en 1870 traza Benjamín Vicuña Mackenna y que justamente el plan de Brunner busca incluir a través de la extensión un anillo de vialidad (Sabatini, Cáceres y Cerda, 2001).

En una fotografía de Karl Brunner de 1932 (Figura 1) se puede ver un tramo recién construido de la Avenida Perú (denominada Avenida de Circunvalación) a la altura de la calle Rapa-Nui. Se observa el perfil de una gran avenida con un bandejón verde central. Un modelo de avenida-parque que Brunner proyectó también para otros sectores de Santiago como Av. Matta o Av. Cumming. Aun cuando, en ese momento la vía tenía solo unas pocas cuadras $(600 \mathrm{~m}$ ) ya que hacia el norte (en la calle San Cristóbal) estaba cortada por las instalaciones de un molino de harina y un Club de Tiro (Pavez Reyes, 2013). 


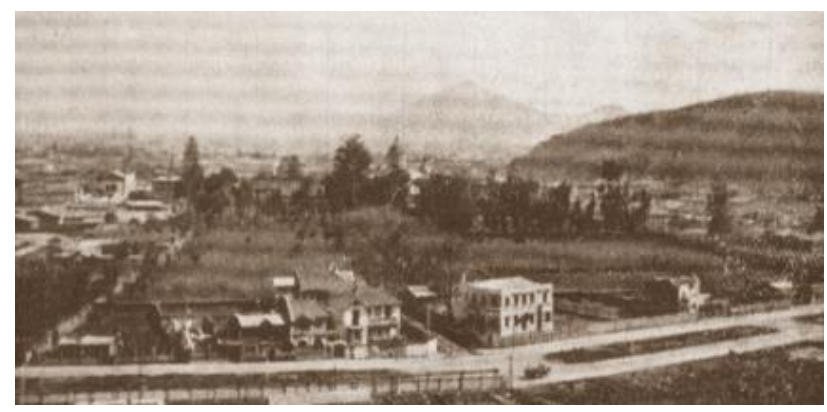

Figura 1. Avenida de Circunvalación. La imagen corresponde a Av. Perú con Rapa Nui. Fuente: Brunner, 1932, p.108.

Como se puede entrever a partir de esta fotografía, existe un contexto que explica el cómo se va gestando esta intervención urbana. En este caso las preguntas que se plantean son: ¿Cuál es el contexto que explica por qué se decide y materializa esta intervención? ¿A quién(es) le interesa la intervención? y ¿Cómo se va definiendo la escala del proyecto?

En primer término, se puede decir que existe un contexto histórico, donde, en este caso, son gravitantes los instrumentos de planificación y los procesos de urbanización.

La Avenida Perú se proyecta, como una idea hacia el futuro, en el Plano Oficial de Urbanización de la Comuna de Santiago, de Karl Brunner 1934, como una avenida que va desde la calle Loreto hasta la Avenida Valdivieso y continúa al norte por el antiguo camino de El Salto, pero aun sin llegar a lo que hoy es Américo Vespucio ( $\underline{\text { Figura }}$ 2). Es el momento de las grandes avenidas haussmanianas como ejes estructuradores de la ciudad.

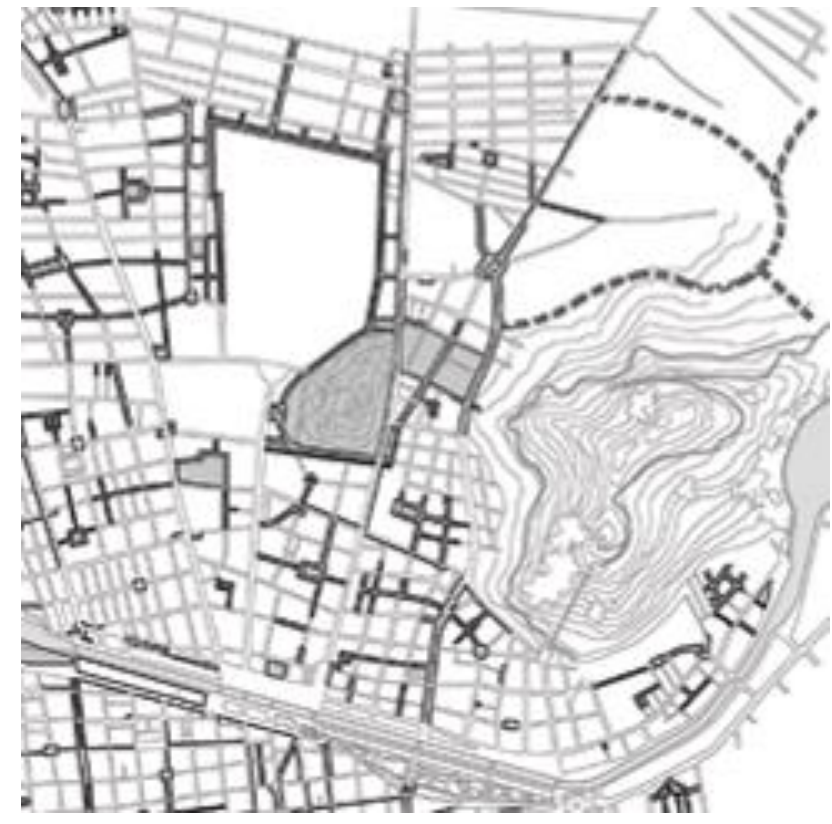

Figura 2. El trazado de Av. Perú en el Plan Regulador de Brunner de 1934. Detalle del Plano oficial de urbanización de la comuna de Santiago, 1934. Fuente: Archivo Municipalidad de Santiago.

Luego en el Plan Regulador Intercomunal de Santiago (PRIS 1960), que se caracteriza por su imaginario de los grandes ejes viales que estructuran la ciudad intercomunal, la avenida pasa a ser parte del eje troncal: el eje Lira-Carmen, Loreto, Av. Perú, El Salto. Uno de los grandes ejes viales que cruzan entera la ciudad de nortesur, para articularse con el anillo de circunvalación Américo Vespucio. Es un eje que implicaba y sigue considerando importantes expropiaciones para su materialización, las que aún no se han podido realizar en su totalidad, pasando en el tiempo por proyectos fallidos ${ }^{1}$ o su construcción por tramos².

Por último, en el Plan Regulador Comunal (PRC) de Recoleta del 2005, un plan regulador que surge de la necesidad de dar unidad e identidad al territorio de la nueva comuna de Recoleta $^{3}$, se considera a la Av. Perú

\footnotetext{
${ }^{1}$ Eje Lira Carmen Loreto, 1997. Proyecto SERVIU que nunca llegó a construirse.

2 Lira-Carmen, proyecto Corredor de Transporte Público, Transantiago, 2009.

3 La comuna había surgido en 1991 de un reordenamiento administrativo de las comunas de Santiago. En este caso el territorio se formó a partir dos antiguas comunas, la parte sur era parte la comuna de Santiago y la norte y oriente de Conchalí.
} 
como parte del eje Lira-Carmen, Loreto, Av. Perú, EI Salto, el eje T6N del PRMS 1994, heredero del PRIS de 1960. Pero, casi como si fuera un comentario al margen, en el cuadro en el cual se describe la vialidad se le agrega al tramo de Av. Perú la condición de vía parque con ciclovía ${ }^{4}$. Esta condición se incorporó al PRC a partir de la demanda y propuesta de las organizaciones ciclistas, que solicitaron incluir en la planificación comunal una red de ciclovías ${ }^{5}$.

Junto a esta definición de la avenida (o la vía) en los instrumentos de planificación, se puede distinguir en el entorno un interesante proceso de urbanización con distintos fragmentos que corresponden a distintos períodos. En la Carta de Santiago de 1918, del Instituto Geográfico Militar (Figura 3) donde aún no existe la calle se pueden observar tres tramos: al sur un sector de chacra agrícola; al centro, en el espacio donde los cerros se aproximan, unas manzanas ya urbanizadas y un gran paño, ambos estructurados en sentido oriente poniente; y hacia el norte, al poniente se grafican manzanas consolidadas y hacia la ladera del cerro chacras agrícolas. Esta división en tres tramos se refleja en los fragmentos de urbanización.

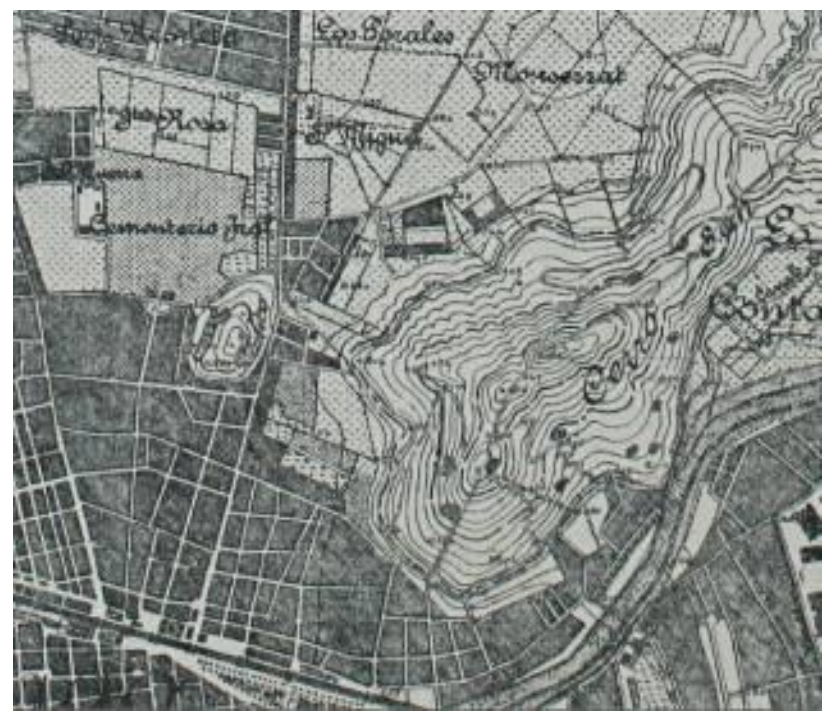

Figura 3. El sector de Av. Perú en Carta de Santiago, 1918, Instituto Geográfico Militar. Fuente: Archivo Biblioteca Nacional.

${ }^{4}$ Cuadro $N^{\circ} 8$, Vialidad estructurante, Municipalidad de Recoleta (2005).

${ }^{5}$ Carta de observaciones del público en el proceso de consulta del proyecto PRC Recoleta 2005, que el proyecto acoge e incorpora.
De sur a norte desde Dominica a Valdivieso se puede distinguir primero el barrio residencial Av. Perú, que corresponde al loteo (hacia 1930) de los terrenos del convento de la Recoleta Dominica y que se caracteriza por grandes casas que pertenecieron a la comunidad árabe-palestina, que primero se había ubicado en el entorno próximo de la calle Patronato. Es el barrio residencial acomodado, ordenado en torno a la avenidaparque que estaba apareciendo en la fotografía de 1932.

Hacia el norte, donde se produce el acercamiento entre los cerros San Cristóbal y cerro Blanco, existió un enclave agroindustrial ubicado en el recorrido del canal de La Pólvora: el molino San Cristóbal, la fábrica de muebles Undurraga, junto a un gran predio que fue el Polígono de Tiro. Este enclave dividió la Av. Perú en dos tramos sin conexión hasta fines de los años 60. Por lo mismo, es un área que vivió un proceso de transformación posterior marcado por la intervención de la CORMU en 1970, a través del gran proyecto de remodelación urbana que fue la Población Polígono de Tiro, luego llamada Población San Cristóbal ${ }^{6}$.

El tramo norte, al que se accedía sólo desde la Av. Valdivieso, corresponde también a un antiguo barrio agrícola-industrial (calles Unión, Puma, María Graham) ubicado en el entorno de los grandes predios del Cementerios General (1821) y el Cementerio Católico (1878). En ese tramo, al poniente de la actual avenida se ubicaba la fábrica textil Mota (hasta aproximadamente el año 2000) y hacia el oriente la población Lemus (loteo 1950).

En este contexto dado por la planificación y el proceso de urbanización, se puede observar que existe un momento particular en que se dan las condiciones para la intervención. Hacia 1990 - 2000 confluyen en este caso tres factores: una dinámica de renovación urbana y densificación en altura; una política pública de apoyo y financiamiento a la intervención en espacio público; y la emergencia de los movimientos ciclistas.

\footnotetext{
${ }^{6}$ La población San Cristóbal, ex Polígono de Tiro, corresponde a uno de los proyectos de remodelación urbana realizados por la CORMU (Corporación de Mejoramiento Urbano) en el período 1966-1976. En la gestión y diseño de este conjunto participó el arquitecto Miguel Lawner, Premio Nacional de Arquitectura 2019.
} 
El primer edificio en altura que se construye en la comuna de Recoleta se encuentra en el sector sur del barrio Avenida Perú. Es un edificio de vivienda, de 14 pisos, construido alrededor de 1990. Este edificio es parte del proceso de renovación urbana en altura y densificación predial que se produce en el centro y pericentro de la ciudad de Santiago a partir de la década de 1990. Según señalan López, Gasic, Meza y Arce (2014), este proceso refleja una fuerte dinámica inmobiliaria que estaría respaldada por cambios normativos como expresión de una gestión urbana proempresarial. En el caso del barrio Avenida Perú los primeros edificios fueron construidos con la normativa proveniente de la comuna de Santiago7y llegan a los 20 pisos, en tanto el cambio normativo restringió la altura a 14 pisos, los que sin embargo se incrementan a través de normas especiales. El dinamismo inmobiliario de Av. Perú sin duda estuvo y está determinado por la calidad ambiental del sector: la vía parque y la proximidad al cerro San Cristóbal. En este escenario el extender la avenida-parque significa también ampliar hacia el norte unas condiciones ambientales favorables a la renovación urbana y la inversión inmobiliaria.

El segundo factor son las políticas urbanas de espacio público. Así como el gobierno de Ricardo Lagos se caracterizó por el fuerte impulso a las concesiones y a las grandes obras de infraestructura y vialidad ${ }^{8}$. En el mismo periodo emerge progresivamente una preocupación por el espacio público, que se manifiesta a través del programa de Recuperación de espacio públicos patrimoniales del MINVU. En la comuna de Recoleta se realizan en el marco de este programa, primero el proyecto Remodelación de la Fray Andresito (2003) y luego el Mejoramiento de la Calle Pío Nono (2005) ubicado a pocas cuadras del inicio sur proyecto. Una intervención que consideraba un paseo y ciclovía.

Otra intervención importante en el área, que se enmarca tanto en la política de espacio público como de protección de la infancia, es la construcción del Parque Bicentenario de la Infancia (2012) ubicado justamente en el tramo central, el entrecerros. Esta intervención, así

\footnotetext{
${ }^{7}$ Zona C PRC de Santiago, 1990.

8 En la comuna de Recoleta: Costanera norte (2000); Metro Línea 2 (2004-2006); Vespucio Norte (2006); Túnel El Salto-Kennedy.
}

como la calle Pio Nono, visibilizan y amplían el área de influencia de avenida desde lo barrial y local a un público que se abre al resto de la ciudad.

El último factor interesante de destacar en este caso es la emergencia de los movimientos pro-bicicleta ${ }^{9}$. Desde la sociedad civil surge a mediados de 1990 y se infiltra hacia las instituciones públicas (municipios, SECTRA, Seremitt, CONASET, UOCT) un grupo de usuarios y profesionales que promueven el uso de la bicicleta y apoyan la creación de una red de ciclovías en Santiago. En ese escenario extender la incipiente ruta de Pio Nono y abrir una red hacia el área norte fue apoyada abiertamente por las organizaciones de ciclistas locales (como Recocleta), pero sobre todo santiaguinas.

En resumen, el contexto que en este caso explica la intervención, es un entramado que va desde la planificación urbana a factores particulares que confluyen en un momento y que generan una sinergia y confluencia de intereses, desde el sector inmobiliario, al sector público y la sociedad civil, que son los que movilizan la intervención urbana.

\section{La gestión desde el nivel local}

Según su definición se entiende por gestión la tarea de "Llevar adelante una iniciativa o un proyecto"10. En esa línea, en esta sección se pretende registrar y revisitar cuál fue el proceso para llevar adelante esta iniciativa: cuales fueron las etapas, que actores resultaron claves y que recursos fueron los necesarios.

La especificidad de la gestión que en este caso, se trata de un proyecto de inversión pública, de escala intermedia, gestionado desde el municipio.

Los municipios, según el Artículo 1 de la Ley Orgánica Constitucional de Municipalidades (2001) son: "corporaciones autónomas de derecho público, (...) cuya finalidad es satisfacer las necesidades de la comunidad local y asegurar su participación en el progreso económico, social y cultural de las respectivas comunas". En ese marco la municipalidad de Recoleta es un

\footnotetext{
${ }^{9}$ Desde 1995 hasta ahora los grupos ciclistas organizan la cicletada del 1er martes del mes.

${ }^{10}$ Fuente: Diccionario Real Academia Española.
} 
gobierno local, con un nivel de acción que por una parte se encuentra muy próximo a las necesidades de la población local, pero por otra, interviene en un territorio que no es solo local sino metropolitano (Orellana, Allard, Néspolo y Mercado, 2012). La avenida Perú es un espacio de barrio, pero al mismo tiempo es un eje metropolitano, por lo tanto, es una vía de paso de los residentes y trabajadores de la zona norte de Santiago. Es la puerta de acceso al Parque de la Infancia, un equipamiento de la ciudad. En este sentido es un espacio local y al mismo tiempo de uso metropolitano.

Por lo mismo, una de las características del proceso fue una progresiva definición de la escala de la intervención. Como se observará más adelante, en un primer momento el alcance del proyecto comprendía 2,1 km (Av. Perú y Valdivieso) y luego en un segundo momento se ajustó a sólo la Av. Perú (1,6km).

Actualmente en el ámbito de las intervenciones urbanas se puede distinguir una variedad de escalas que van desde la planificación al urbanismo táctico, pasando por los grandes proyectos urbanos (Lange Valdés, 2014), obras de infraestructura o los programas de mejoramiento de barrios. En Recoleta, en los últimos años se han realizado grandes obras de infraestructura, como el Corredor de transporte público Av. Dorsal (Transantiago, SERVIU, \$35.000.000.000) o inversiones en espacios públicos de barrios, en el marco del programa Quiero mi barrio. Por ejemplo, Barrio Venezuela: (SERVIU, M. de Recoleta, 400-700 millones de pesos). Entre estos rangos el proyecto de Mejoramiento
Av. Perú se puede decir que por el monto corresponde a una escala intermedia (M. de Recoleta, \$3.000.000.000), pero que interviene un eje intercomunal y que por lo tanto involucra la revisión y aprobación de instancias del nivel central.

A su vez el monto de inversión excede ampliamente las posibilidades financiamiento con recursos municipales, por lo que una primera decisión de la gestión municipal fue abordar la etapa de diseño (aproximadamente $\$ 40.000 .000$ ) con recursos propios y postular a fondos de nivel regional (FNDR) para la ejecución (aproximadamente \$3.000.000.000).

\section{Un panorama de las etapas y tiempos del proyecto}

En la metodología de evaluación de proyectos de inversión pública se distinguen 4 etapas: idea, diseño, ejecución y operación. En este caso la idea parte el 20062007 con algunas imágenes objetivo, fotomontajes, presentaciones e intervenciones provisorias, bastante similares a lo que hoy se denomina urbanismo táctico (Fernández Prajoux, 2016). Ideas que son un preámbulo a la etapa de diseño, que fue claramente la etapa más extensa (8 años aprox.). Esto comparado al tiempo de ejecución, que entre la gestión de los recursos, licitación y la construcción propiamente tal fue de alrededor de 2 $1 / 2$ años (Figura 4). En este sentido parece interesante revisar justamente como se explica el largo proceso del diseño. 


\begin{tabular}{|c|c|c|c|c|c|c|c|c|c|c|c|c|c|}
\hline $\begin{array}{c}\text { Nombre del } \\
\text { proyecto }\end{array}$ & Etapa & Año & 2008 & 2009 & 2010 & 2011 & 2012 & 2013 & 2014 & 2015 & 2016 & 2017 & 2018 \\
\hline & IDEA & & & & & & & & & & & & \\
\hline \multirow{4}{*}{$\begin{array}{c}\text { Area verde y ciclovía } \\
\text { Av. Perú }\end{array}$} & DISEÑO & & & & & & & & & & & & \\
\hline & LICITACION 1 & & & & & & & & & & & & \\
\hline & LICITACION 2 & & & & & & & & & & & & \\
\hline & DISEÑO 1 & & & & & & & 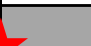 & & & & & \\
\hline \multirow{5}{*}{$\begin{array}{c}\text { Mejoramiento } \\
\text { espacio público Av. } \\
\text { Perú }\end{array}$} & DISEÑO 2 & & & & & & & 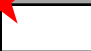 & & & & & \\
\hline & EJECUCION & & & & & & & & & & & & \\
\hline & FINANCIAMIENTO & & & & & & & & & & & & \\
\hline & LICITACION & & & & & & & & & & & 7 & \\
\hline & CONSTRUCCION & & & & & & & & & & & J & \\
\hline \multirow{2}{*}{\multicolumn{2}{|c|}{$\begin{array}{l}\text { Cambio de período alcaldicio } \\
\text { Re-elección }\end{array}$}} & & & & \multicolumn{5}{|c|}{ Cambio período presidenial } & & & & \\
\hline & & & & & & & & & & & & & \\
\hline \multicolumn{2}{|c|}{ Inauguración } & & & & & & & & & & & & \\
\hline
\end{tabular}

Figura 4. Cronograma de las etapas del proyecto. Fuente: Elaboración propia, 2019.

La etapa de diseño parte con dos licitaciones. La primera coincide con el final de un período alcaldicio, lo que puede explicar un cambio de prioridades que lleva a que la licitación se declare desierta, aun cuando existían 3 ofertas. La segunda en tanto corresponde al inicio de otro periodo alcaldicio y lleva efectivamente a contratar a un equipo consultor que se va a mantener durante todo el largo proceso de diseño y hasta el seguimiento de la obra.

Entre el 2010 y el 2013 nos encontramos con una primera etapa en el diseño, en donde lo que estaba programado para un período de aproximadamente un año, dividido en anteproyecto, proyecto y aprobaciones, se detiene $y$ extiende en el momento de las aprobaciones $^{11}$.

Al analizar el proceso de esos años se puede concluir que se presentaron 3 nudos críticos. Al inicio de las revisiones y aprobaciones surgió la dificultad de que la consultoría (por bases) no consideraba cambios en la semaforización. La falta de estos estudios fue cuestionada por las unidades de transporte de nivel municipal y nivel central. Para resolver el problema la municipalidad debió contratar directamente un consultor

\footnotetext{
${ }^{11}$ Estas involucran la revisión y aprobación del proyecto por parte de las distintas instancias municipales y sobre todo de las unidades técnicas de nivel central: específicamente Seremitt, MINVU, Transantiago, SECTRA, SERVIU, CONASET, UOCT.
}

de la especialidad, lo que implicó un retraso por tiempos de contratos y una complejidad en la coordinación entre 2 consultores.

Un segundo nudo correspondió a los cambios políticos y técnicos asociados al cambio de gobierno el 2010. Durante el año 2011 se produce un cambio de los cuadros técnicos y un proceso de revisión y reformulación del funcionamiento de las instancias ligadas al transporte y en particular de los lineamientos para la construcción de ciclovías.

Por último, a fines del 2012, cuando los estudios de especialidades y aprobaciones ya estaban en una etapa final, el avance del proyecto coincide con el cambio de alcalde. El nuevo alcalde Daniel Jadue, de profesión arquitecto y sociólogo, de ascendencia palestina y antiguo vecino del barrio Av. Perú, por una parte, decide continuar con la iniciativa, pero también hacer un cambio importante al diseño. Hasta ese momento el diseño incluía un primer tramo con parque central y luego un segundo tramo en el cual se ampliaban las veredas. El alcalde con un objetivo de equidad territorial toma la decisión de que el proyecto debe ser unitario: en todo el recorrido un bandejón central con césped, que extienda la calidad ambiental y urbana del antiguo barrio de grandes casonas de la Av. Perú hacia el norte, hacia el sector más pobre. A partir de esa decisión respalda la continuidad del equipo consultor y los equipos técnicos municipales. 
Con estos nuevos lineamientos el proyecto se reformula y al mismo tiempo cambia de nombre, iniciándose una nueva etapa de diseño que se realizó entre el 2014-2015.

Es significativo el cambio de nombre, de Área verde y ciclovía Av. Perú a Mejoramiento espacio público Av. Perú, ya que por una parte refleja un cambio en la decisión política y a su vez un giro en las políticas públicas, que se refleja en el criterio de formulación frente al Ministerio de Desarrollo Social. Una iniciativa que parte enfocada a soluciones parciales (o sectoriales en el lenguaje de la evaluación social de proyectos) deriva a una solución integral (o multisectorial).

\section{Complejidad, sinergia y participación}

El giro de lo sectorial a lo multisectorial representa una de las características importantes para entender la gestión de este proyecto. Lo multisectorial es una categoría cada vez más utilizada en evaluación de proyectos públicos y está asociada a la compresión de los problemas urbanos no como un asunto de parcialidades, especialidades o sectores. El problema de intervenir una calle no es sinónimo de vialidad o de construir una ciclovía o un área verde, sino que supone asumir un enfoque próximo a los sistemas complejos.

Si se analiza este caso, la complejidad está dada por las distintas especialidades que componen el proyecto y a la vez por los distintos niveles involucrados. Como en toda obra, en este caso confluyen varias especialidades: arquitectura, paisajismo, pavimentación, señalización y demarcación, semaforización, alumbrado y riego. Al mismo tiempo la experiencia fue el proyectar, revisar y tomar decisiones en conversaciones a distintos niveles: los arquitectos y especialistas dentro del equipo consultor, la contraparte y las distintas unidades municipales, la comunidad local y de ciclistas, y luego los profesionales de ministerios y servicios centrales. Esta amplitud se expresa en el número de profesionales que participaron en la etapa de diseño: 11 profesionales por parte de la consultora y 23 por parte del municipio, de 6 unidades (Tabla 1).

\section{Tabla 1}

Cuadro de profesionales

\section{Municipalidad de Recoleta}

Secpla y Asesoría Urbana

Mariela Aranda (Encargada etapa diseño 1), Paulina Ahumada (Encargada etapa diseño 2). Gianinna Repetti, Catherine Manríquez, María José Rioseco, María Rene Noguera, Pablo Figueroa, Patricio Flores, Juan Luis Aravena.

\section{Dirección de Tránsito}

Juan Veliz, Ariel López.

\section{Dirección de Obras}

Alfredo Parra, Pablo Vázquez, Jorge Ardiles, Mauro Rodríguez, Fanny Salazar, Pamela Vergara, Marco Bustamante.

\section{Dirección de Medio Ambiente y Ornato}

Francisco Moscoso, Patricia Castañeda.

\section{Dirección de Desarrollo Comunitario}

Fares Jadue, Alejandro Martínez.

\section{Dirección de Deporte}

María Belén Fernández.

\section{Consultora}

Arquitectura y paisaje

Juana Zunino, Paz Carreño.

\section{Colaboradores}

Laura Font, Pablo Rosen, Claudio de la Cerda, Rosario Palacios.

Ingeniería

Argia Ingenieros: Jorge Albornoz, María Victoria Amigo, Gabriel Muñoz.

\section{Semaforización}

Macro Ingeniería: Rodrigo Díaz, Luis Inostroza.

Fuente: Elaboración propia, 2018. 
Durante el año 2014, al inicio de la aprobación del proyecto por parte de la Seremi de Transporte, que trabaja como una ventanilla única, donde confluyen en una mesa los representantes de todas las instancias relacionadas con transporte a nivel metropolitano, se discutió por casi 6 meses la opción de pasar de un ancho de pista vehicular de 3,5 a $3 \mathrm{~m}$. Esto no se puede leer como un problema de burocracia, sino una negociación sobre cómo se distribuye el espacio público: desde la mirada centrada en la vialidad, inscrita en la Ordenanza general de urbanismo y construcciones, OGUC, a las reivindicaciones de las organizaciones ciclistas y propeatones, que promueven una redistribución más equitativa del espacio público. Esta negociación, en este caso impulsada desde un nivel intermedio como es el del municipio, habría sido inviable sin la colaboración o sinergia entre los profesionales de los distintos niveles y en particular la transversalidad con que se venía dando la promoción de las políticas pro-ciclistas, que se había traducido en el Plan Maestro de Ciclovías para Santiago (Sectra, 2013).

Por último, el otro aspecto relevante de la gestión del proyecto tiene que ver con los procesos de participación ciudadana. En este caso la consultoría consideraba la realización de talleres de participación.

En la primera fase de diseño se realizaron 2 talleres y varias reuniones. En este momento fueron especialmente activos los grupos ciclistas, que representaban no el segmento barrial sino el de los usuarios metropolitanos.

En la segunda fase se realizaron 2 talleres en los que participaron vecinos residentes, dirigentes, empresario y representante del Parque Metropolitano. La opción desde el municipio fue realizar las reuniones en las sedes vecinales de las poblaciones Lemus y San Cristóbal, para incentivar justamente el compromiso con los residentes del tramo norte de la avenida. Adicionalmente se realizaron otras 2 reuniones en terreno, específicamente en las plazoletas ubicadas en los conjuntos de vivienda social. Estas porque como eran espacios comunes de uso público y se visualizó en las reuniones generales que era necesario una instancia de información y conversación particular con los vecinos directamente involucrados. Intervenir estas "áreas café" fue una opción de equidad territorial y a su vez un espacio de conflicto, ya que involucraba conciliar los intereses particulares sin perder la condición de un uso público.

En definitiva el proceso de participación jugó un papel en distintos planos: el diseño se fue alimentando de las necesidades, preferencias y significados locales; se fue levantando un grupo de actores interesados en que se materialice la iniciativa y que a la larga resultan claves en la continuidad de la iniciativa; y por otra parte, la discusión entre distintos actores con distintos intereses, a veces contrapuestos, va construyendo un cierto espacio de civilidad, un espacio de acuerdos y convivencia, que busca expresarse en el diseño mismo.

\section{El proyecto de espacio público: un desafío de diseño integral e integración urbana}

Se observan nuevos giros de lo sectorial a lo complejo; de la vialidad, e incluso de la movilidad, hacia un enfoque más integral de espacio público en la política pública. Ejemplo de ello se manifestó en la visita del arquitecto danés Jan Gehl a principios del 2016 invitado por el Ministerio de Vivienda Urbanismo (MINVU) y el Gobierno de la Región Metropolitana.

Tanto en la intervención en el sector de Mapocho y la publicación por parte del MINVU de una guía para el análisis y diseño de espacios públicos (MINVU, PNUD y Gehl, 2017) se observa una mirada integral y por otra, un enfoque no hacia los vehículos, ya sean autos o bicicletas, sino hacia los usuarios. Diríamos hacia la "humanización del espacio público" que postula Gehl (2006). En una de las reuniones de participación ciudadana, un activo ciclista y jefe técnico del Parque Metropolitano, lo expresaba así: "Esto no es un proyecto de ciclovía, este es un parque".

En lo medular, el proyecto propone conciliar la complejidad de situaciones en una propuesta unitaria, armónica y funcional, con un lenguaje claro para el usuario y con espacios flexibles que acojan la diversidad de actividades a lo largo del año.

En este marco el proyecto de Av. Perú, desde Domínica hasta Valdivieso, se entiende como una unidad espacial, destacando los elementos comunes a lo largo de la avenida, como son el contexto geográfico y cultural. Ellos han conformado la imagen interna que los habitantes conservan en la memoria colectiva y que el 
diseño se propone develar en una aproximación a la naturaleza y a la identidad con el lugar.

El diseño resume e incorpora básicamente los conceptos integradores entre ciudad y naturaleza, propuestos por distintos autores desde el ámbito de la arquitectura del paisaje (Laurie, 1975; McHarg, 1992; Hough, 1998 y Careri, 2002).

\section{Contexto geográfico y cultural}

En el contexto geográfico, la Av. Perú se ubica en el borde poniente del cerro San Cristóbal, hacia el norte del río Mapocho. Entre el cerro San Cristóbal y el cerro Blanco ( Figura 5). La presencia permanente del cerro marca el carácter del lugar, en una relación cercana y constante con la naturaleza. La mole del cerro se levanta por el oriente, con una fuerte pendiente que sirve de respaldo natural, abriendo las vistas lejanas hacia el poniente (Felsenhardt, Cáceres y Zunino, 2002).

La imagen de la ladera de color tierra conserva vestigios de la vegetación nativa semiárida, de espinos silvestres y arbustos, mezclados con una vegetación arbolada mixta de colores verdes matizados, con especies de clima mediterráneo (Zunino, 2003).

Como se observa en el mismo grabado, en la relación con la ciudad patrimonial, este sector ha sido el acceso tradicional o paso obligado desde el norte hacia el centro del valle de Santiago en la época del asentamiento prehispánico y luego de la llegada de los españoles, a la ciudad fundacional.

Esta condición ha sido enriquecida a través de la historia, con una serie de edificios públicos emblemáticos que son los umbrales de entrada o salida a este barrio. Desde el centro histórico, los accesos, a través de tres puentes, se identifican con la Escuela de Derecho de la Universidad de Chile, el Museo de Bellas Artes, el Mercado Central, y la estación Mapocho.

A ellos se agregan otros hitos patrimoniales del barrio como son los cementerios tradicionales, las iglesias coloniales y los barrios de fuerte identidad como Patronato, con sus tiendas típicas, la Vega Central, con los productos para la alimentación y Bellavista, con los cafés, restoranes y discoteques que la caracterizan.

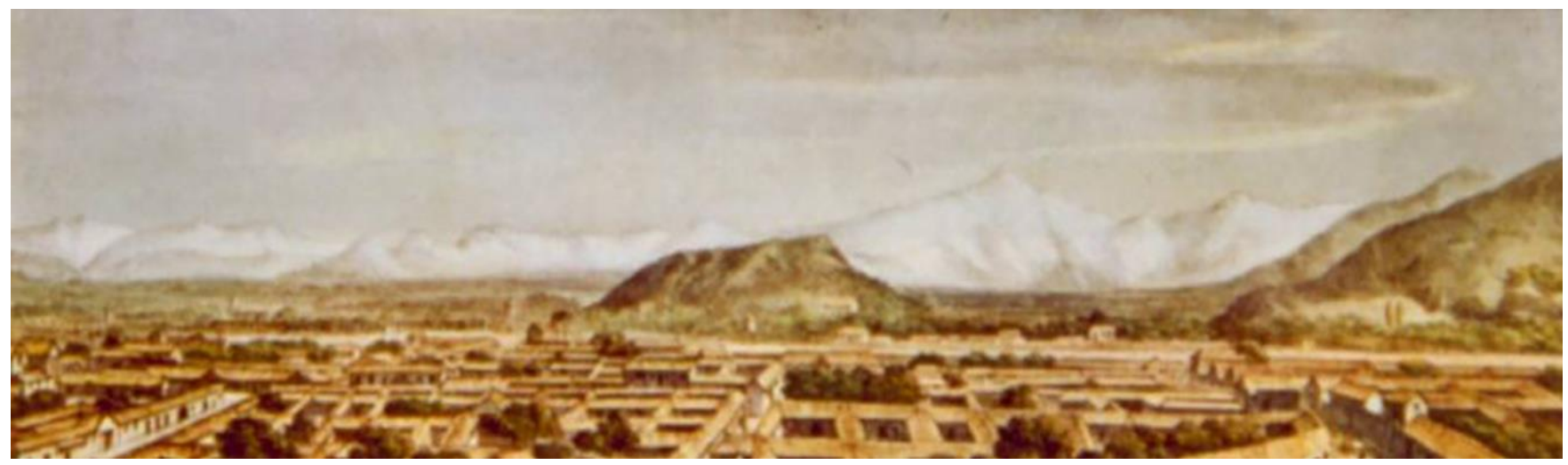

Figura 5. Vista de Santiago. Waldegrave 1831. Fuente: Felsenhardt, Cruz, Maino y Zunino (2000). 


\section{Sistema de áreas verdes}

La Av. Perú, será parte del sistema de áreas verdes central de la ciudad, conectada con la Plaza Italia y el río Mapocho, donde convergen los parques de Borde río, como el Parque Forestal y el Parque Providencia, el Parque Bustamante hacia el sur, los Cerros islas, representados por el Cerro Santa Lucía y el Cerro Blanco y el cerro San Cristóbal que conecta con la cordillera al oriente.

Es parte de un conjunto de piezas, que un estudio en curso para la Región Metropolitana denomina "Mosaico ecológico urbano" y se propone relevarlas como elementos claves de una planificación ecológica y sustentable de la región (Picon, M., De la Barrera, F., Contreras, C., Fernández, I., Berrizbeitia, A. y ReyesPaecke, S., 2017).

En este caso, el carácter de corredor verde, facilitado por la ubicación relacionada a elementos geográficos relevantes como el río Mapocho y el cerro San Cristóbal, permite ser parte de un sistema ecológico que atrae aves e insectos que colaboran en la descontaminación de la ciudad.

El corredor verde se va construyendo en el tiempo gracias a la presencia de árboles añosos de gran envergadura, como plátanos orientales en los cabezales, desde Domínica y Valdivieso, que son las huellas de un pasado rural, en la periferia de la ciudad y los ceibos, contemporáneos a las edificaciones de los años 50-60.

\section{Descripción del proyecto}

La propuesta de arquitectura: un parque lineal. El conjunto de factores que comprende desde el contexto geográfico y cultural a la voluntad política se transforman en la base de las decisiones del diseño.

La prolongación del bandejón central de $600 \mathrm{~m}$ a $1.600 \mathrm{~m}$ cambia el carácter de la calle, generando un parque lineal, con una dimensión que en el largo, contiene un paseo peatonal, ciclovía, una cinta lúdica para los niños y franjas laterales con vegetación. El ancho que fluctúa entre 9.00 y 5.00 m permite desarrollar pausas, a lo largo del paseo en una secuencia de estaciones para diversas actividades: lugares de encuentro, fuentes de agua, pérgolas y un circuito de máquinas de ejercicio. En el conjunto se pueden desarrollar distintas actividades, aportando lugares temperados con la sombra de los árboles, la humedad ambiental proporcionada por las fuentes de agua, el colorido estacional de las flores y las sonoridades del follaje con la brisa a distintas horas del día.

Se puede reconocer que una de las decisiones claves del diseño fue la cinta lúdica. El objetivo era realizar una propuesta unitaria, reconociendo que existían 3 tramos, diferentes a lo largo de la avenida, que era necesario unificar (Figura 6).

Los tramos se diferencian por el carácter de los edificios, alturas, año de construcción, materialidad, usos y vegetación existente. Se vuelve relevante constatar que esos 3 tramos son los que se distinguían en el plano de 1918. 

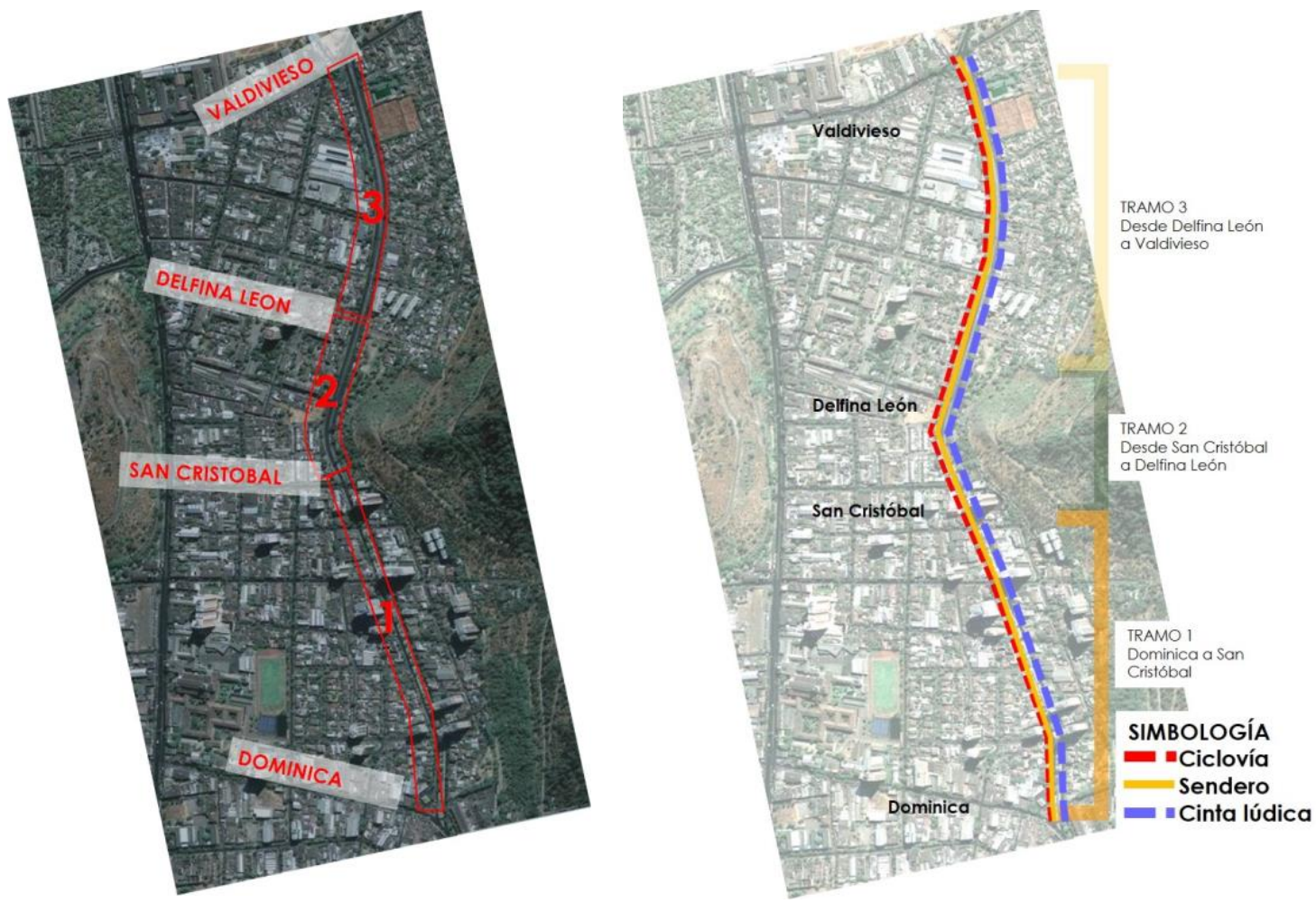

Figura 6. 3 tramos distintos y 3 elementos de continuidad. Fuente: Elaboración propia a partir de imagen Google Earth, 2018.

La cinta es un elemento constante multifuncional, que sirve de contención, de asiento y de juego lineal, con lugares para los distintos usuarios: ciclistas, deportistas, vecinos y peatones. Su forma dinámica en pendientes diversas, a escala menor, se asimila al paisaje de cerros circundantes

La dificultad original, originada en un bandejón angosto donde resultaba difícil ubicar zonas de juegos seguras para niños, se transformó en que finalmente son los niños, a través de la cinta lúdica, los que dan unidad al proyecto. No es casual que la cinta se convirtió en la imagen del proyecto que se usó por ejemplo en las invitaciones a los talleres de participación (Figura 7 y Figura 8.1).

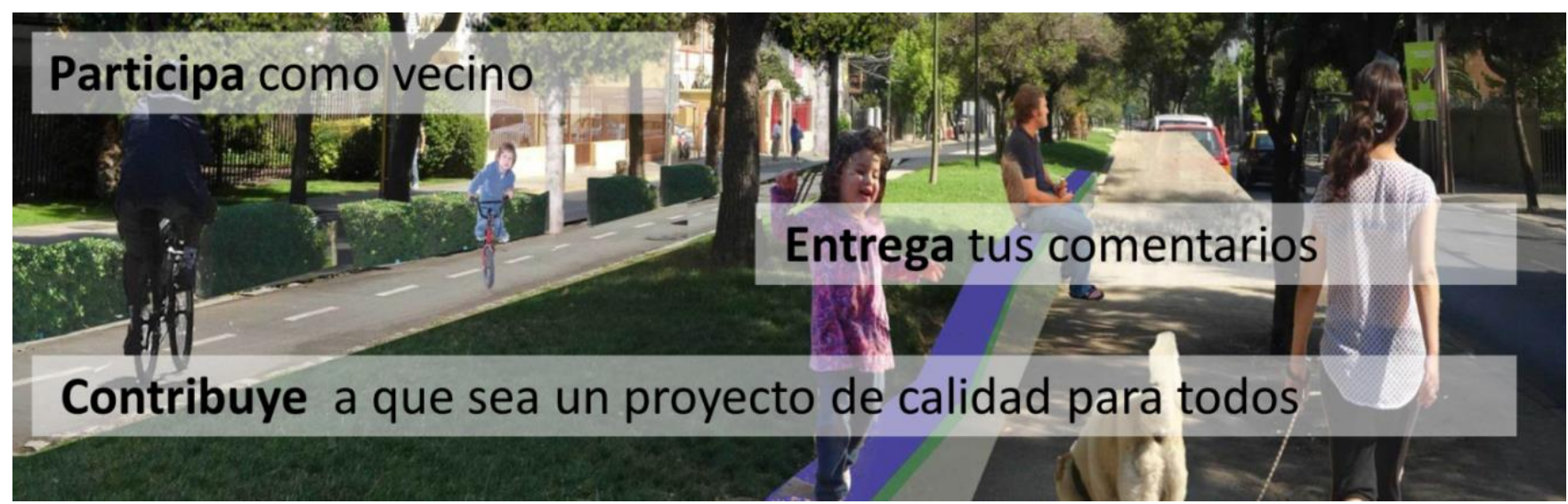

Figura 7. Invitación a talleres de participación. La cinta lúdica como imagen central del proyecto. Fuente: Memoria proyecto Mejoramiento espacio público Av. Perú. Municipalidad de Recoleta, Zunino, J. et al. (2015). 


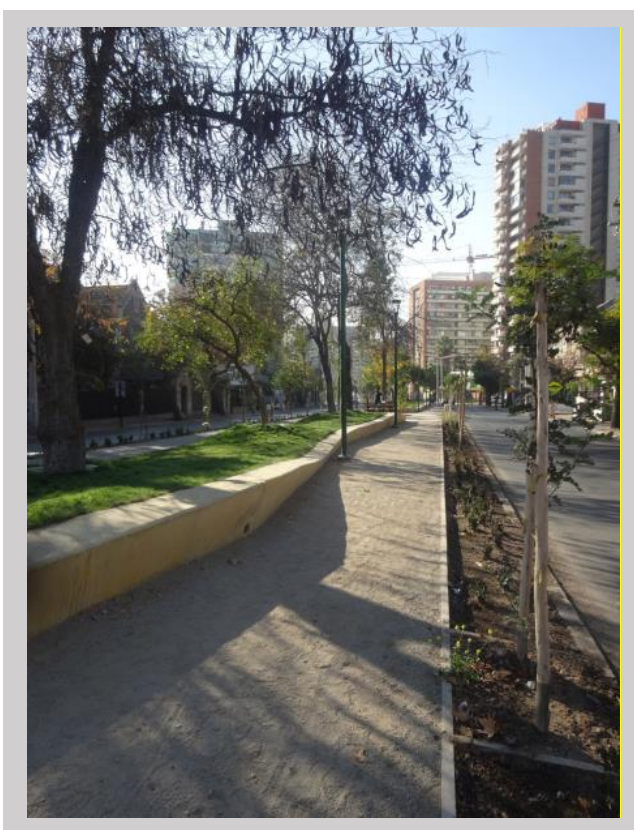

8.1 La cinta lúdica multifuncional entre el jardín central, el sendero peatonal y la platabanda con árboles.

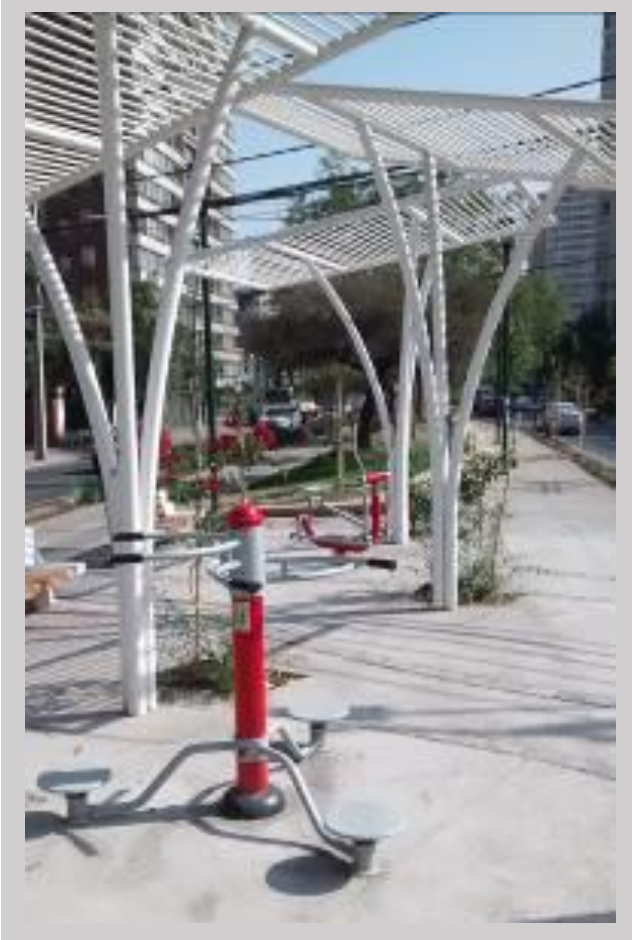

8.3 Sombreaderos con enredaderas

y máquinas de ejercicio

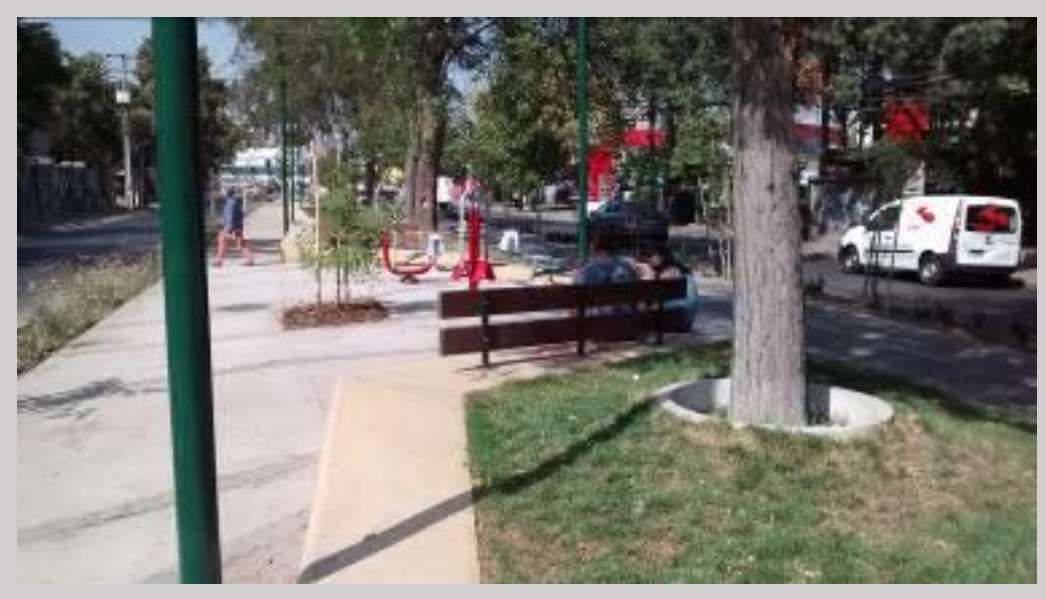

8.2 El paseo lineal y estaciones de descanso con sombrederos

o arboladas, circuito de máquinas de ejercicios.

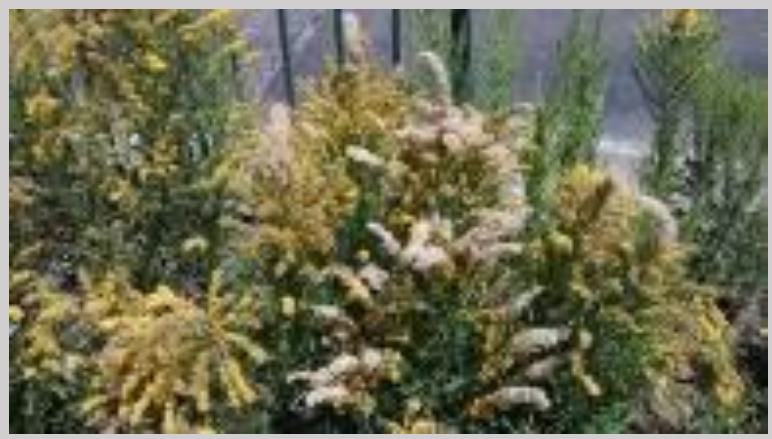

8.4 Flora nativa: Solidago chilensis, florecido y con abejas.

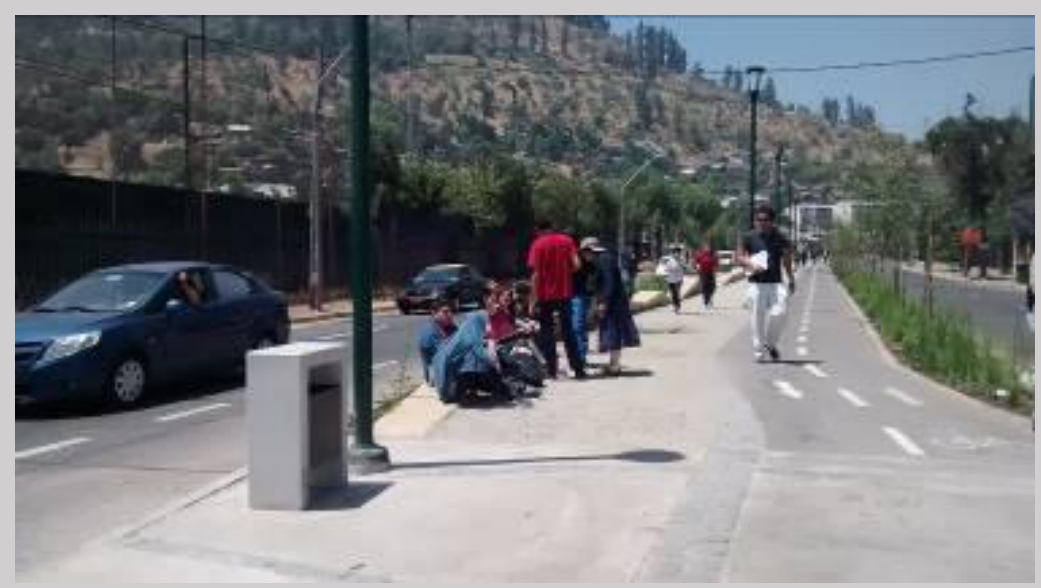

8.5 Clases de educación física, escuela Valentín Valdivieso,

en el nuevo Parque lineal Av. Perú.

Figura 8. La unidad dada por la cinta lúdica, espacios abiertos de encuentro en las intersecciones, flora nativa y apropiación social del espacio. Fuente: fotografías de los autores. 
En el tramo central, la vecindad de conjuntos habitacionales con espacios privados de uso público, como las plazoletas, han permitido ampliar las áreas verdes relacionando los espacios exteriores aledaños, que pueden formar futuros corredores verdes transversales en la ciudad, especialmente en el sector denominado entrecerros (Pavez Reyes, 2013; Municipalidad de Recoleta, 2005).

Otra definición importante del diseño es que los cruces de calles se han jerarquizado según el carácter del entorno. Se han privilegiado las vistas hacia el cerro con los hitos existentes (La Virgen, Parque de la Infancia, el ex molino, etc.) y las vistas a edificios significativos hacia el poniente (iglesias, etc.) conformándose como miradores despejados para peatones y automovilistas (Figuras 8.2 y 8.3).

\section{El paisajismo: una apuesta por la flora nativa y mediterránea}

El proyecto de paisajismo se ha concebido en forma conjunta con los de arquitectura y especialidades, reforzando su funcionalidad, caracterizando los lugares y conformando a la vez, una unidad espacial armónica, a través de una arborización reconocida en el recorrido y relacionada con la vegetación cercana.

La selección de la vegetación se basa en los estudios sobre flora nativa y mediterránea realizados por Beytía et al. (2012), Gajardo (1995), Gildemeister (2006), Hoffmann (1982, 1983), Luebert y Pliscoff (2006), Niemeyer y Teillier (2007), Riedemann y Aldunate (2001), Rodríguez y Quezada (1982) y Saldías (2011).

La materialidad de los elementos construidos y la selección de la vegetación responden a un concepto de calidad y permanencia en el tiempo considerando los atributos de los recursos naturales, sus condiciones climáticas, temperatura, humedad ambiental, viento, la disponibilidad de agua de riego y de buenos sustratos naturales y las posibilidades de una real mantención en las instituciones a cargo, considerando su sostenibilidad a mediano y largo plazo. Se incorpora además a la comunidad como un actor activo y responsable en el cuidado del lugar.

Se valoriza la presencia de árboles añosos que representan una época en la ciudad, como los ceibos, plátanos orientales y las especies nuevas como jacarandas, palmera fénix y brachychiton en veredas remodeladas. Los algarrobos europeos, pimientos y vilcas se rescatan de plazas y lugares circundantes.

Por otro lado, la cercanía con el cerro y las condiciones originales del paisaje de Santiago motivan a una recuperación de las especies nativas del bosque esclerófilo, aportando beneficios al medio ambiente a través de la densificación de las especies de follaje persistente y las asociaciones naturales que atraen insectos y aves.

Es así como el quillay se constituye en el árbol que acompaña a la ciclovía en el bandejón central, protegiendo del sol de la tarde, separando de la calzada, marcando un ritmo permanente en toda su longitud.

Los lugares significativos se reafirman con especies nativas como palmas o bellotos y en las veredas se continúa con los celtis australis y plátanos existentes. Ello va entregando diversidad de formas y colores que alegran y orientan en el recorrido.

En la plaza cívica central, que se ubica en el entrecerros, el lugar de acercamiento entre el cerro San Cristóbal y el cerro Blanco, frente al Parque de la Infancia, se especifica palma chilena, para recuperar una especie emblemática del país, dar significado especial al sector y con una relación visual lejana libre, entre sus troncos-pilares.

Por último, la propuesta paisajística se cierra como una unidad entre árboles, arbustos y cubresuelos, en asociaciones que aportan color en diferentes épocas del año, formas, texturas, aromas diversos y versatilidad en las formas y en la mantención de alturas apropiadas al carácter del lugar (Figura 8.4).

\section{Objetivos y recomendaciones de diseño para nuevos espacios públicos de envergadura}

Como primeras conclusiones se puede decir que la experiencia chilena nos indica que, para la materialización de un espacio público de envergadura, se requiere un cierto período de años y la coordinación de una gran complejidad de temas y objetivos.

En base a la experiencia del proyecto de Avenida Perú, el presente artículo presenta una pauta a modo de 
hoja de ruta, de modo de incorporar desde el inicio de la formulación de un proyecto de espacio público el sinnúmero de aspectos que debe incluir, junto a una sintética guía de recomendaciones de diseño de arquitectura y paisaje (Tabla 2 ).

\section{Tabla 2}

\section{Objetivos y recomendaciones de diseño para nuevos espacios públicos de envergadura}

Objetivo Recomendaciones

\section{Tema: Gestión}

Generar una sinergia positiva entre el equipo mandante y el equipo de diseño (arquitectos e ingenieros)

Promover la continuidad en el tiempo de los equipos de trabajo.

Dar continuidad al proyecto a pesar de las condiciones políticas imperantes a nivel local (municipio) como nacional (presidencia)

Incorporar desde el inicio de la licitación o formulación del proyecto la complejidad de aspectos técnicos

\section{Tema: Criterios de diseño arquitectónico}

Integración social para generar mayor equidad Inclusión de la historia y la identidad del lugar

Generar inclusión de una gran diversidad de usuarios: niños, jóvenes, adultos, ancianos, personas con capacidades espaciales (minusválidos, ciegos, etc.)
Generar un ambiente de confianza mutua en las decisiones profesionales, respeto responsabilidad y flexibilidad

Mantener equipos de trabajo, tanto en el mandante como equipos de diseño, en las etapas de anteproyecto, proyecto y supervisión en la ejecución

Ubicar al usuario como el objetivo central de la construcción del espacio público, cualquiera sea la tendencia de las autoridades de turno

Incluir la totalidad de temas requeridos en un espacio público según su escala: arquitectos, ingenieros (aguas Iluvias, cálculo estructural, calzadas; semaforización etc.
Elección de materiales nobles para dar continuidad en el largo plazo al proyecto

Generar espacios públicos espacialmente continuos que relaciones barrios de diversos grupos Diseñar incorporando elementos de identidad cultural y/ o patrimonial que vinculan a los habitantes con el lugar

Diseñar incorporando:

- Elementos de juego para niños, en este caso un recorrido de color para saltar, subir, bajar o sentarse, en un contexto protegido y seguro (con barrera de plantas o metálica cercana a la calzada)

- Espacios de encuentro multietario, con diferentes usos según las horas del día o las estaciones: plazoletas, asientos, fuentes de agua, máquinas de ejercicio, ciclovía, sendero, miradores, etc.

- Para jóvenes: áreas de césped, ciclovía y espacios de asientos

- Para adultos: máquinas de ejercicios, senderos peatonales. Ciclovía, Lugares de estar.

- Para adultos mayores: Asientos en una distancia mínima de 200 m, con sombra o sol.

- Diseñar bajo las normas de accesibilidad universal definidas en la normativa vigente, incorporando rampas en semáforos y cruces, línea de baldosas para guía de ciegos, entre otros.

Estimar los recursos económicos totales y diseñar en base a ellos, de modo de incorporar elementos urbanos que perduren en el tiempo (al menos 20 años) y sean antivandálicos. Ejemplo: asientos de hormigón visto con sello antigraffiti, diseño de asientos de hormigón en obra con color incorporado en la mezcla, luminarias altas y a prueba de piedrazos, uso de adoquines de piedra para diferenciar sectores. Incorporar gran cantidad de áreas de suelo permeable (maicillo, etc.).

\section{Tema: Criterios de diseño paisajístico}

Dar continuidad a la mantención del proyecto.

Es importante coordinar este aspecto con el Departamento de Parques y Jardines o de Medio Ambiente de la Municipalidad correspondiente, o el organismo que hará la mantención.

Aportar a la biodiversidad local

Aportar a la descontaminación de partículas Incorporar especies de identidad local
- Proponer especies de bajo consumo de agua y baja mantención (podas, etc.), adecuadas al clima local. En la zona central de Chile, se especificarán especies de clima mediterráneo, de Temuco al sur, se especificarán especies de zonas húmedas y frías.

- Proponer especies de árboles que no dañen veredas en el largo plazo con sus raíces

Integrar flora nativa y especies con flores adaptadas al clima local; definiendo previamente la región vegetacional de cada caso.

No incorporar especies invasoras o eliminar las existentes, por no permitir la biodiversidad. Maximizar la propuesta de árboles persistentes, generando avenidas que acompañen calzadas y bosquetes en el interior de plazas y parques Incluir especies que se observen en las cercanías del proyecto y donde exista una vinculación emocional y formen parte de la memoria colectiva. Ej.: jazmines por su aroma, etc.

Fuente: Elaboración propia, 2018. 


\section{Conclusiones}

Al plantearse la revisión de este caso el objetivo central fue registrar cómo se desarrolló el proceso y como se pueda aprender de él y mejorar en el caso de intervenciones similares. Si nos apoyamos en la idea de que los "factores (que intervienen) pertenecen a la esfera de lo técnico, lo institucional, lo político y lo social" (Echeverri y Orsini, 2011, p.21), proponemos aquí hacer el recuento en esos términos.

En la esfera de lo técnico un primer aprendizaje, que repercutió en los tiempos fue no haber considerado una especialidad (en este caso justificación de semáforos). En este sentido al revisar el proceso nos encontramos con un progresivo ajuste en la formulación del problema y la definición de la escala de intervención. Fue estratégico el enfoque hacia un diseño integral. A su vez, acotar el problema también fue un paso necesario. La primera versión de diseño consideraba 2 problemáticas distintas: una era Av. Valdivieso y el acceso a los cementerios y otra la Av. Perú con sus distintos tramos. El proceso llevó a abordar solo una y adecuarse así a las posibilidades de la gestión desde lo local.

En la misma esfera de lo técnico un factor clave en este caso fue la continuidad del equipo consultor a lo largo de todo el proceso y su participación hasta la supervisión de la obra. La obra urbana como vimos es producto de un conjunto de especialidades e instancias de decisiones, pero mantener la coherencia del diseño hasta la construcción, es un tema de autoría necesario para lograr una obra urbana de calidad y no una suma de intervenciones inconexas.

En la esfera de lo institucional un aprendizaje central fue, que al igual que con las especialidades del proyecto, es necesario considerar desde el inicio la coordinación con todas las unidades involucradas dentro del municipio y en los distintos servicios del nivel central. Pero, la coordinación es necesaria pero no suficiente. En este caso se puede decir que la clave estuvo en la sinergia: en una actitud de colaboración entre los organismos del estado en pro de dos lineamientos estratégicos de la política pública: el espacio público y el uso de la bicicleta.

En cuanto a la esfera política, es evidente y ya conocido que la voluntad de la autoridad política es el primer paso de una obra urbana. Los tiempos y cambios de los periodos presidenciales y alcaldicio son un factor determinante, como se evidencia en este proceso en las inflexiones en los años 2008-2010-2012. En esta experiencia el giro del proyecto fue producto de la voluntad política, una voluntad que al mismo tiempo fue estratégica en mantener al equipo consultor y respaldar la gestión del equipo técnico municipal.

Para terminar, respecto del factor social, lo particular del caso como se pudo ver, es que se trata de una iniciativa de gestión local, pero en un espacio metropolitano, lo que significa una articulación en estos 2 niveles: el barrio y la ciudad. El interés metropolitano, expresado en los movimientos ciclistas por impulsar la construcción de una red de ciclovías en la zona norte de la ciudad, resultó clave en la continuidad del proyecto, por su presencia y presión en los niveles centrales del estado.

A la vez el nivel local, expresado en los grupos de los barrios, los dirigentes de las juntas de vecinos, los vecinos de las plazoletas, es el ámbito social propio de la gestión municipal. Fue un factor que alimentó la identidad del proyecto, a través de los talleres de participación, reuniones y durante la construcción. No estuvo exento de conflictos, pero son estos usuarios los que le dan sentido y proyección a la intervención. La obra urbana es un momento, los profesores y estudiantes de la escuela Valentín Valdivieso que se apropian del espacio para hacer las clases de educación física ( Figura 8.5), son parte de la construcción social del espacio, un proceso donde será interesante una evaluación más adelante [R] 


\section{Referencias}

Beytía, A. et al. (2012). Guía de arborización urbana. Especies para la RM. Santiago: CIEDESS

Brunner, K. (1932). Santiago de Chile. Su estado actual y futura formación. Santiago: Imprenta La Tracción.

Busquets, J. (2011). Modelos de proyecto urbano. En Montaner, J., Álvarez, F. \& Muxi, Z (Eds.) Archivo Crítico modelo Barcelona (1973-2004). Barcelona: Ajuntament de Barcelona, Departamento de Composición Arquitectónica ETSAB/UPC.

Careri, F. (2002). El Andar como Práctica Estética. Barcelona: Editorial G. Gilli.

Echeverri, A. y Orsini, F. (2011). Informalidad y urbanismo social en Medellín. Sostenible?, (12), 11-24. Disponible en http://www.eafit.edu.co/centros/urbam/articulospublicaciones/Documents/111103 RS3 AEcheverri \%20P\%2011-24.pdf

Fernández Prajoux, V. (2016). Acción a corto plazo, cambios a largo plazo. Revista de Arquitectura, 21(31), 78-79. https://doi.org/10.5354/0719$\underline{5427.2016 .44232}$

Felsenhardt, C., Cáceres, G. y Zunino, J. (2002). Informe final del proyecto La imagen del crecimiento de Santiago en la mirada visionaria de los pintores. S. XVI-XX. Proyecto DIPUC, 2001-2002.

Felsenhardt, C., Cruz, Maino y Zunino, J. (2000). Informe final del proyecto La creación del paisaje de Chile. Fondecyt $N^{\circ} 1980$ 587, 1998-2000.

Gajardo, R. (1995). La vegetación natural de Chile. Santiago: Editorial Universitaria.

Gehl, J. (2006 [1971]). La humanización del espacio urbano. Barcelona: Reverté.

Gildemeister, H. (2006). Su jardín mediterráneo. Barcelona: Editorial Moll.

Hoffmann, A. (1982). Flora silvestre. Zona Central. Santiago: Editorial Fundación Claudio Gay.

Hoffmann, A. (1983). El árbol urbano. Santiago: Editorial Fundación Claudio Gay.

Hough, M. (1998). Naturaleza y Ciudad. Barcelona: Editorial G. Gilli.
Lange Valdés, C. (2014). Perspectivas estratégicas y miradas tácticas: propuesta de un enfoque reflexivo en torno al desarrollo de grandes proyectos urbanos. Revista de Urbanismo, (30), 30-38. https://doi.org/10.5354/0717-5051.2014.30887

Laurie, M. (1975). Introducción a la Arquitectura del Paisaje. Barcelona: Editorial G. Gili.

Ley Orgánica Constitucional de Municipalidades N¹8.695, 2001.

López, E., Gasic, I., Meza, D. y Arce, I. (2014). Renovación urbana y rol municipal pro-empresarial en la planificación territorial: Santiago de Chile 1990-2012. Revista Iberoamericana de Estudios Municipales, 8 109-150. Disponible en http://repositorio.uchile.cl/handle/2250/145534

Luebert, F. y Pliscoff P. (2006). Sinopsis Bioclimática y Vegetacional de Chile. Santiago: Editorial Universitaria.

McHarg, I. (1992). Proyectar con la Naturaleza. Barcelona: Editorial G. Gilli.

MINVU, PNUD y Gehl (2017). La dimensión humana del espacio público. Santiago de Chile: Ministerio de Vivienda y Urbanismo. Disponible en http://www.minvu.cl /incjs/download.aspx?glb cod nodo $=201110071235$ 11\&hdd nom archivo=La-dimension-humana.pdf

Moris, R. (2009). Las tuyas, las mías y las nuestras. Ideas para gestión urbana integrada sustentable. Revista $C A,(141)$.

Municipalidad de Recoleta (2005). Plan Regulador Comunal de Recoleta.

Municipalidad de Recoleta, Zunino, J. et al. (2015). Memoria proyecto Mejoramiento espacio público Av. Perú.

Niemeyer, O. y Teillier, S. (2007). Aromas de la Flora Nativa de Chile. Santiago: Editorial Universidad de Chile. 
Orellana, A., Allard, P., Néspolo, R. y Mercado, J. (2012). Gestión urbana municipal a escala metropolitana: modelos en competencia. Revista de geografía Norte Grande,

(51),

67-80.

https://dx.doi.org/10.4067/S071834022012000100004

Pavez Reyes, M. (2013). Un corredor verde entre cerros desde 1934, y las posibilidades actuales desde un ejercicio académico de pregrado en Recoleta. Revista de Urbanismo, 75-95. https://doi.org/10.5354/0717-5051.2013.30306

Picon, M., De la Barrera, F., Contreras, C., Fernández, I., Berrizbeitia, A. y Reyes-Paecke, S. (2017). Resumen ejecutivo final del proyecto Mosaico urbano ecológico: criterios para la planificación ecológica de Santiago.

https://dx.doi.org/10.13140/RG.2.2.22764.46726

Reyes, S. y Figueroa, I. (2010). Distribución, superficie y accesibilidad de las áreas verdes en Santiago de Chile. EURE (Santiago), 36(109), 89-110. https://dx.doi.org/10.4067/S025071612010000300004

Riedemann, P. y Aldunate, G. (2001). Flora Nativa. Chile, Zona Centro. Santiago: Editorial Andrés Bello.

Rodríguez, A. y Sugranyes, A. (2004). El problema de vivienda de los "con techo". EURE (Santiago), 30(91), 53-65. https://dx.doi.org/10.4067/S025071612004009100004

Rodríguez, J. y Arriagada, C. (2004). Segregación Residencial en la Ciudad Latinoamericana. EURE (Santiago), 30(89), 5-24. https://dx.doi.org/10.4067/S025071612004008900001

Rodríguez, M. y Quezada. (1982). Flora Arbórea de Chile. Universidad de Concepción.

Sabatini, F., Cáceres, G. y Cerda, J. (2001). Segregación residencial en las principales ciudades chilenas: tendencias de las tres últimas décadas y posibles cursos de acción. EURE (Santiago), 27(82). https://doi.org/10.4067/s0250-71612001008200002

Sabatini, F. y Brain, I. (2008). La segregación, los guetos y la integración social urbana: mitos y claves. EURE (Santiago), 34(103), 5-26. https://dx.doi.org/10.4067/S025071612008000300001

Sabatini, F., Mora, P. y Polanco, I. (2013). Control de la segregación socio-espacial: Rebatiendo mitos, construyendo propuestas. Espacio Público, 7 Documento de trabajo.

Sabatini, F. y Vergara, L. (2018). ¿Apoyo a lugares o apoyo a personas? Dos proyectos chilenos de vivienda socialmente integrada. Revista INVI, 33(94), 9-48. https://dx.doi.org/10.4067/S071883582018000300009

Saldías, G. (2011). Jardinería en Chile. Santiago: Editorial Universidad Central.

Salinas, E. y Baeriswyl, S. (2017). El Programa de Recuperación Urbana Ribera Norte; veinte años de aciertos y desaciertos de una política de proyectos urbanos en Chile. Revista de Urbanismo, (36), 114130. https://doi.org/10.5354/0717-5051.2017.45676.

SECTRA (2013). Análisis y Evaluación del Plan Maestro de Ciclovías del Gran Santiago.

Zunino, H. (2005). Construyendo ciudad desde lo local en lo global: el caso del proyecto Ribera Norte, Concepción, Chile. Scripta Nova, IX(194). Disponible en http://www.ub.es/geocrit/sn/sn-194-86.htm

Zunino, J. (2003). La evolución histórica del cerro San Cristóbal y la ciudad de Santiago. Revista Jardín Botánico Chagual. Año I, Nº1. 